2nd International Conference on Applied Research in ENGINEERING SCIENCE \& TECHNOLOGY 18-20 October $2019 \quad$ Budapest, Hungary

\title{
The dominant mode shapes of the viaduct-like structures subjected to the passage of high-speed train
}

\author{
Salih Demirtaş ${ }^{1, *}$, Hasan Ozturk ${ }^{1}$ \\ ${ }^{1}$ Department of Mechanical Engineering, Dokuz Eylul University, 35397, Buca, Izmir, Turkey
}

\begin{abstract}
In this study, the mode shapes dominate the dynamic response of a viaduct under the passage of the high-speed train. The viaduct is modelled as a multi-bay frame and the train is modelled as a two-axle multi-body system with 4 DOF. The finite element method with Lagrange's equation has been used to determine the equations of in-plane motions, based on the Bernoulli-Euler beam theory. The Wilson-theta time integration scheme is employed to determine the dynamic response of the structure. To determine which modes are dominate the dynamic response, 3D frequencyvelocity-amplitude graph is plotted.
\end{abstract}

Keywords: viaduct; mode shape; the finite element method; the Wilson-theta method; moving train 